Article

Subscriber access provided by Warwick University Library

\title{
Optimization of the Electrochemically Generated Luminescence of Polyfluorene Films
}

Manuel G. Ramirez, María Angeles Díaz-García, and Francisco Montilla

J. Phys. Chem. C, Just Accepted Manuscript • DOI: 10.1021/acs.jpcc.7b10613 • Publication Date (Web): 30 Jan 2018

Downloaded from http://pubs.acs.org on February 4, 2018

\section{Just Accepted}

"Just Accepted" manuscripts have been peer-reviewed and accepted for publication. They are posted online prior to technical editing, formatting for publication and author proofing. The American Chemical Society provides "Just Accepted" as a service to the research community to expedite the dissemination of scientific material as soon as possible after acceptance. "Just Accepted" manuscripts appear in full in PDF format accompanied by an HTML abstract. "Just Accepted" manuscripts have been fully peer reviewed, but should not be considered the official version of record. They are citable by the Digital Object Identifier (DOI®). "Just Accepted" is an optional service offered to authors. Therefore, the "Just Accepted" Web site may not include all articles that will be published in the journal. After a manuscript is technically edited and formatted, it will be removed from the "Just Accepted" Web site and published as an ASAP article. Note that technical editing may introduce minor changes to the manuscript text and/or graphics which could affect content, and all legal disclaimers and ethical guidelines that apply to the journal pertain. ACS cannot be held responsible for errors or consequences arising from the use of information contained in these "Just Accepted" manuscripts. 


\section{Optimization of the Electrochemically}

\section{Generated Luminescence of Polyfluorene}

\section{Films}

Manuel G. Ramírez ${ }^{1}$, María A. Díaz-García ${ }^{1}$, Francisco Montilla*,2

${ }^{1}$ Dpto. Física Aplicada and Instituto Universitario de Materiales de

Alicante, Alicante 03080, Spain

${ }^{2}$ Dpto. Química Física and Instituto Universitario de Materiales de

Alicante, Alicante 03080, Spain

*corresponding author: francisco.montilla@ua.es 
Abstract

Electrochemically-generated chemiluminescence (ECL) from solid-state poly $\left(9,9^{\prime}-\right.$ octylfluorene) films was obtained by the successive electrochemical injection of polarons with opposite sign (annihilation ECL) in double potential steps. The processes involved in the recombination of polarons, the quenching of the electrochemically generated excitons and the emission stability have been also analysed. A systematic study at various electrochemical potentials has been carried out to determine optimal conditions to obtain a maximum ECL intensity, particularly with potentials of $0.4 \mathrm{~V}$ beyond the doping onsets (for both $p$ and $n$ doping). Results show that while the ECL emission is slightly affected by the potential of the emission, it is strongly dependent on the charging potential. Under large charging potential the electrochemically generated excitons formed in the emission step get quenched by an excess of polarons and bipolarons that are in detrimental of ECL. The maximum emission is obtained when the applied potentials are $0.4 \mathrm{~V}$ over the doping onset of the polymer conjugated chain. After partial depletion of the excess of polarons, the emission coming from the recombination of balanced polarons of opposite sign is observed. The stability of ECL emission in the optimal range was determined in continuous cathodic-anodic pulses. A rapid loss of the intensity in the initial stages is observed due to the dissolution of low molecular weight polymer fragments, that become soluble upon electrochemical doping. The emission spectrum remains stable during the complete lifetime of the polymer. The spectral shape of PFO films indicates that the emission occurs mainly from the $\beta$-phase domains in which the electrochemical charge is preferentially injected. 


\section{Introduction}

Conjugated polymers are very interesting photonic materials for their use in a wide variety of applications such as electronics ${ }^{1}$, lasers and photonics, ${ }^{2-4}$ sensors, ${ }^{5,6}$ lightemitting diodes (LEDs), ${ }^{7-9}$ solar cells, ${ }^{10-12}$ due to their high luminescence quantum yield in solid state and tunable charge transport properties.

Fluorene-based conjugated polymers (polyfluorenes) present some remarkable properties being poly(9,9-dioctylflorenone), PFO, possibly the most widely investigated. PFO is an efficient blue emitter that exhibits a complex morphological behaviour which is highly sensitive to the processing conditions. The solid state structure of PFO presents several phases which possess different inter-monomer torsion angle. ${ }^{3,13-17}$ In the glassy phase, the chains have random orientation resulting in broadened absorption spectra with unresolved vibronic structure. PFO presents other crystalline phases $^{15}, \alpha$ and $\beta$ phases, being the later a very well-known conformation, where the chains adopt an extended and planar zigzag conformation with a torsion angle of $180^{\circ} .{ }^{13,18-20}$ The absorbance spectrum of $\beta$-phase PFO shows a well resolved $0-0$ peak around $435 \mathrm{~nm}$, red-shifted with respect the major peak of the glassy phase. ${ }^{18,21}$ For all phases, the photoluminescence (PL) spectrum exhibits a vibronic structure with red-shifted peaks from glassy ${ }^{18,22}$ to $\beta$-phase. ${ }^{15}$ This later phase dominates the emission even when it is only present in a small fraction in the sample, due to fast migration of excitons from glassy to the lower energy $\beta$-phase domains. ${ }^{21,23,24}$ The good optical and electrical properties of $\beta$-phase PFO make it an ideal candidate for electroluminescent devices such as organic light-emitting diodes (OLED), electrically pumped organic lasers or light-emitting electrochemical cells (LEC). ${ }^{25,26}$ The latter device is based in the phenomenon of electrochemically generated luminescence or 
electrochemiluminescence (ECL). In those devices the excitons are generated by the recombination of electrochemically generated charge carriers (polarons) with opposite charge, in a process known as annihilation ECL. ${ }^{27}$ Annihilation ECL has attracted considerable interest for its potential in the development of sensors and novel luminescent devices. ${ }^{28-33}$ However, annihilation ECL of conjugated polymers is still a relatively unexplored proccess. ${ }^{34}$ The major reason is the anomalous properties observed in the electrochemical doping switching of conjugated polymers, mainly because the injection of charges in the conjugated chain induces the simultaneous intercalation of ions coming from the electrolyte. ${ }^{35,36}$ The basic annihilation ECL mechanism can be represented by four steps: (i) charge injection, (ii) transport and recombination of polarons of opposite charge, (iii) exciton formation and (iv) radiative decay of excitons. ${ }^{37,38}$ A key aspect for ECL optimization is the selection of suitable potentials to generate a maximum amount of emitted light. The number of injected charges must be balanced, since the doping at low potentials generates a small number of carriers leading to low emission intensity. On the other hand, the use of very large potentials for doping promote the formation of species that may be harmful for luminescent emission as bipolarons. ${ }^{37}$ A detailed study of this process would be useful for the prospect of developing electrochemically pumped solid state lasers. The idea of a laser operating by ECL was proposed in the $1970-\mathrm{s}{ }^{39,40}$. Such a device would be of great interest because it would not require an additional source for optical pumping, besides other advantages, such as easy wavelength tunability or possible miniaturization. Horiuchi et al. showed the possibility of obtaining amplified emission in an electrochemiluminescent cell containing a conjugated dye. ${ }^{41}$ The cell had semitransparent platinum electrodes forming the resonant cavity. When the redox 
process was performed in the cell, an intensified emission was observed with a

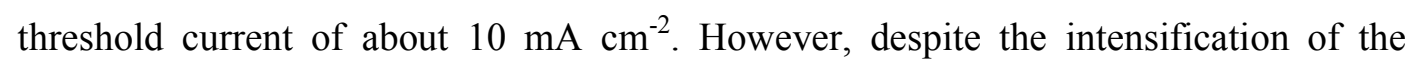
emitted light, which follows a superlineal behavior with the pumping energy, no other characteristics of the amplified emission were observed. An important problem of this type of design is the low mobility of the luminescent reagents that must diffuse from the bulk solution to the surface of the electrode where they react. Under these conditions the electrochemical reactions are limited by the diffusion coefficient of the redox active species.

The annihilation ECL process of a solid-state polymer presented in this work allows the injection and accumulation of charge carriers of near the electrode-polymer interface, so that the low mobility of the carriers in these polymers would no longer be an issue for exciton accumulation. The first step for the development of this type of devices requires an optimization of the electrochemical conditions in order to achieve a high concentration of excitons that would allow population inversion.

In the present work, we report ECL obtained from the annihilation process of PFO films. A detailed study of the dependence of the ECL intensity on the anodic and cathodic potentials, aiming to obtain a maximum emission efficiency has been carried out. The processes involved in the recombination of polarons, the quenching of the electrochemically generated excitons and the emission stability have been also analysed. Such a study constitutes a first essential step towards the development of ECL lasers.

\section{Experimental part}


PFO end capped with dimethylphenyl groups was purchased from American Dye Source (reference ADS129BE, $\mathrm{M}_{\mathrm{W}}=40,000$ - 150 000). The chemical structure is shown in the inset of figure 1 . PFO thin films were drop-casted from a $6 \mathrm{mg} / \mathrm{mL}$ solution in chloroform (Sigma Aldrich, anhydrous) over fluorine-doped tin oxide electrodes (FTO, SOLEMS, AGC 80, 70-90 ohms). Prior to their use, these electrodes were rinsed with ethanol.

The solvent used for electrochemical measurements was anhydrous acetonitrile (Merck Seccosolv) and 0.1 M tetrabutylammonium hexafluorophosphate (Sigma-Aldrich, for electrochemical analysis) was used as supporting electrolyte.

Electrochemical measurements were performed in a modified fluorescence cell $(1 \mathrm{~cm}-$ length quart cell, design showed in the supporting information). The modified fluorescence cell was covered with reflective layer composed of a mixture of white paint and $\mathrm{BaSO}_{4}$, leaving an uncovered $1 \mathrm{~cm}^{2}$-window to recollect the emission beam.

The working electrode was a transparent FTO glass covered with PFO thin film. Pt and Ag wires were used as counter and pseudo-reference electrodes, respectively. The reference electrode was calibrated with the ferricenium/ferrocene couple $(E=0.64 \mathrm{~V}$ vs SHE) ${ }^{42}$. The oxygen was purged from the electrochemical cell by bubbling an argon flow for 10 min and the argon atmosphere was maintained during all the experiments. Electrochemical experiments were performed making use of a function generator (EG\&G Parc 175 model) connected to a potentiostat-galvanostat (Pine instrument AFCBP1 model).

Photoluminescence (PL) and electrochemiluminescence (ECL) spectra were acquired at $15{ }^{\circ} \mathrm{C}$ using a PTI QuantaMaster spectrofluorometer (QM-62003SE model) and an Ocean Optics spectrophotometer (Flame model). 


\section{Results and discussion}

Figure 1 shows the absorbance (ABS) and PL spectra of a PFO film drop-casted over a FTO electrode at room temperature. The ABS spectrum shows a peak at $437 \mathrm{~nm}$, related to $0-0$ vibronic transition of $\beta$-phase PFO whereas the peak at $395 \mathrm{~nm}$ corresponds to absorption from both, the $\beta$ and glassy PFO phases ${ }^{43}$. The amount of beta phase can be determined from UV-vis experiments and in the present case there was determined to be $10 \%$. The fraction of repeat units was determined by subtracting the glassy film absorbance from the measured absorbance after normalization at $354 \mathrm{~nm} .{ }^{44}$ The PL spectrum measured at an excitation wavelength of $365 \mathrm{~nm}$ shows a vibronic progression with peaks at 441,468 , and $500 \mathrm{~nm}$ assigned to the $\mathrm{S}_{1} \mathrm{~S}_{0}$ singlet exciton transitions. This spectrum is characteristic of the emission of the $\beta$-phase PFO. ${ }^{18,45}$ The low energy peak with maximum at $538 \mathrm{~nm}$ is attributed to emission from fluorenone defects and to the formation of dipole mediated stacking molecular in solid state ${ }^{46-48}$.

Typical stabilized cyclic voltammograms of the PFO thin film deposited on an FTO electrode is shown in the inset of figure 1 . The PFO polymer can be reversibly $\mathrm{n}$ and $p$ doped. The oxidation and reduction onsets were $1.02 \mathrm{~V}$ and $-2.10 \mathrm{~V}$, respectively. 


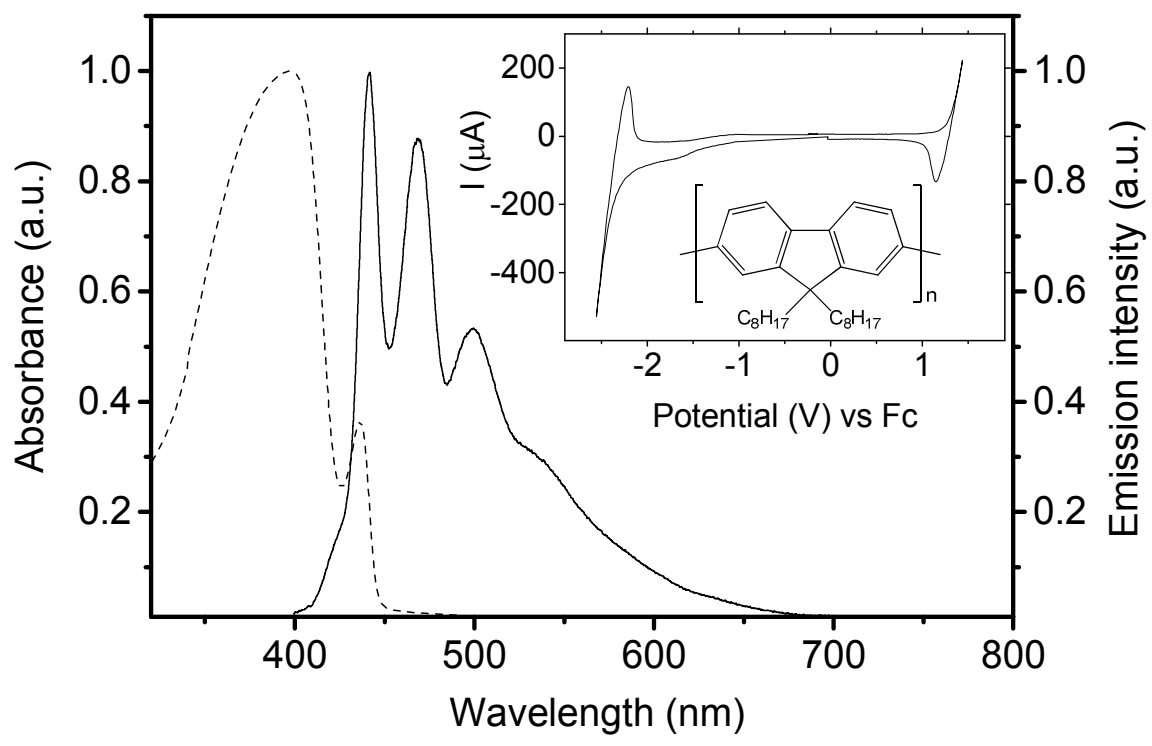

Figure 1. PL (solid line) and ABS (dashed line) spectra of a PFO film deposited by drop-casting from chloroform solutions over FTO electrodes. Inset: stabilized cyclic voltammogram obtained in the study of reversible doping processes of a PFO thin film with a scan rate of $100 \mathrm{mV} \mathrm{s}^{-1}$ and chemical structure of PFO.

The electrochemical experiments for the ECL emission were performed by double potentiostatic step. In a set of experiments, the potential was initially stepped to a negative value to proceed to the $n$-doping of the polymer (charging stage). After that the potential was stepped to a positive value for producing $p$-doping. The emission is produced during this anodic step by the recombination of the $n$-polarons existing in the film with the new injected positive charges. We call this process anodic emission (AE).

Similar experiments were performed by applying initially a positive potential for the $p$ doping in the charging stage and the emission is produce in the step to negative values of potential, we named this process cathodic emission (CE). 


\subsection{Anodic emission}

We first studied the ECL emission from the PFO films obtained during anodic pulses (see figure 2).

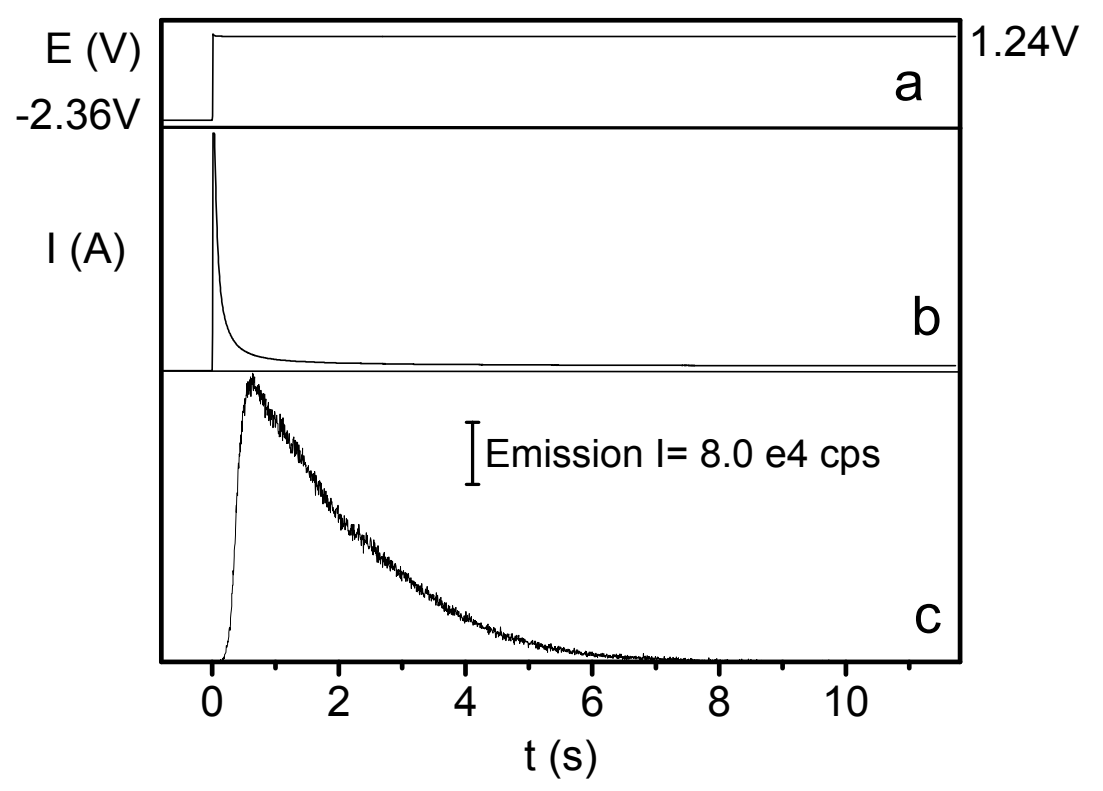

Figure 2: (a) Potential applied to the working electrode; (b) Current intensity registered during the potentiostatic step; (c) Emission intensity of recorded at $440 \mathrm{~nm}$. Working electrode FTO/PFO electrode in a solution acetonitrile + 0.1M TFB-TBA.

The electrochemical potential was initially fixed to a cathodic value, chosen to be more negative than the reduction onset of the polymer $(E=-2.36 \mathrm{~V}$, fig. $2 \mathrm{a})$ to proceed with the injection of electrons (electrochemical $n$-doping) and the formation of $n$ polarons for a certain period, achieving the charge saturation of the conjugated chain (when the current reached a value of 0 , equation 1$)$ :

$$
S_{0}+e^{-} \rightarrow p^{-}(\text {saturation })
$$


Then, the potential was stepped to an anodic potential more positive than the oxidation onset of the polymer $(\mathrm{E}=+1.24 \mathrm{~V})$. It drives to electrochemical injection of holes $(\mathrm{p}-$ doping). As observed from $\mathrm{t}=0 \mathrm{~s}$, a positive current is detected which corresponds to the charge and the injection of $p$ polarons in the conjugated chain (fig. $2 \mathrm{~b}$, equation 2 ):

$$
S_{0}-e^{-} \rightarrow p^{+}
$$

The polarons with opposite sign recombines with the formation of polaron pairs that form singlet excitons (see equation 3), responsible of the emission (equation 4):

$$
\begin{aligned}
& p^{+}+p^{-} \rightarrow S_{1} \\
& S_{1} \rightarrow S_{0}+h v
\end{aligned}
$$

Figure 2.c shows the emission intensity measured at a wavelength of $440 \mathrm{~nm}$ during the anodic pulse. After a "dark period" of $0.15 \mathrm{~s}$ during which no emission is detected, the ECL starts to grow until reaching a maximum intensity at $0.60 \mathrm{~s}$. Then, the ECL intensity decays until reaching a value the half of its initial value (half-life time) after $1.6 \mathrm{~s}$. The ECL is completely extinguished $9 \mathrm{~s}$ after the emission starts.

The shape of the ECL transients cannot be fitted by a simple decay function, indicating that the electrochemiluminescence process is a combination of a complex process happening in the polymer film. This issue will be analysed in detail in subsequent sections.

A systematic study on various potentials for anodic ECL (Anodic Emission, AE) was performed. The charging potential was fixed to a value of $-2.36 \mathrm{~V}$ to saturate the conjugated chain with negative polarons (eq. 1). Figure 3.a shows the ECL transients for some of the anodic potentials used, ranging from 1.04 to $1.64 \mathrm{~V}$, to inject positive polarons (eq. 2). 

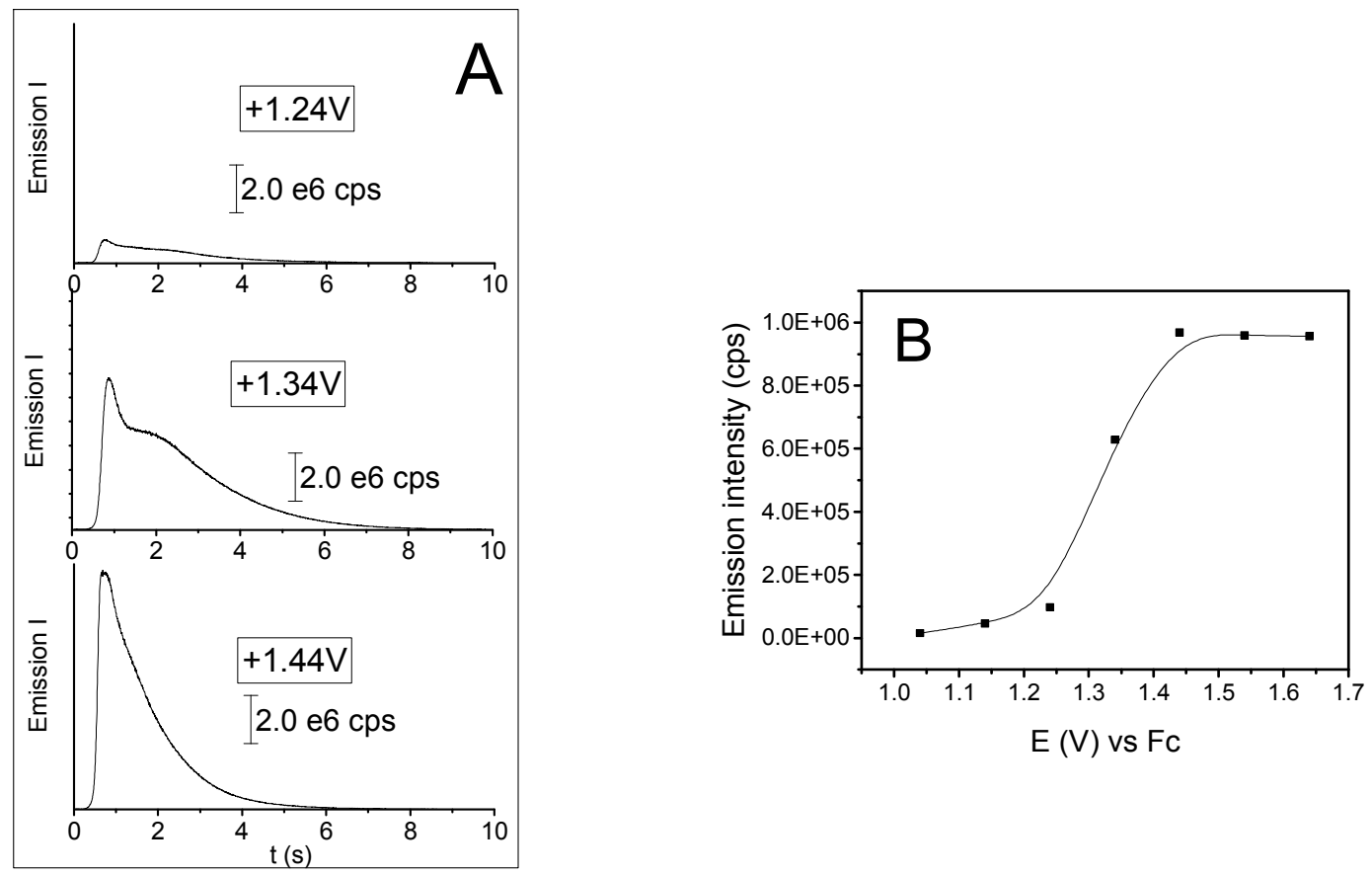

Figure 3. A) Intensity of the emitted light at $\lambda=440 \mathrm{~nm}$ in an anodic pulse (AE) at different potentials (indicated in each graph). B) maximum ECL intensity at $\lambda=440 \mathrm{~nm}$ at different anodic potentials. The charging cathodic potential was fixed at $-2.36 \mathrm{~V}$.

As observed in fig. 3A, initially no emission is observed up to near 0.4-0.6 s after the potential step: Then light emission appears, reaching a maximum intensity at about $1 \mathrm{~s}$ after the step, due to the recombination of polarons and exciton decay. The half-life time is around 1.5-2.5 $\mathrm{s}$ and there is no correlation between this time and the emission potential. The intensity decreases and a complete vanishing of ECL is observed at about 7-9 s after the potential step. The ECL transients show complex shapes with shoulders between 2 and $5 \mathrm{~s}$. The shapes of the ECL transients may be indicative of the presence of charge trapping defects (as monosubstituted fluorene moieties or fluorenone 
moieties) on the charge transport and recombination. During the charge at negative potential, some negative charges may be trapped in mono-substituted defects and aromatic cyclopentadienide anion are formed. Further, the aromatic radical may react with close polymer chains originating crosslinking. ${ }^{38}$ Thus, these defects seem to give rise to trapping effects in both, $n$ and $p$ polarons and consequently to other emission mechanisms or quenching.

With regards to fig. $3 \mathrm{~B}$, the maximum intensity increases slightly from $+1.04 \mathrm{~V}$ (close to the $p$-doping onset) until $+1.24 \mathrm{~V}$, potential at which a strong increase of the ECL emission occurs. When the potential reaches $+1.44 \mathrm{~V}$, the AE maximum intensity remains stable although it slightly decays at higher potentials.

When the anodic potential is $+1.44 \mathrm{~V}$, the probability to obtain emissive recombination between $n$ and $p$ polarons is close to the maximum and, then, the highest AE intensity is measured.

The nature and number of charges injected in the polymer is controlled by the applied potential. ${ }^{49,50}$ The quenching of the excitons by polarons and the radiative decay are competitive process and depend on the number of charges injected. The number of holes injected during the anodic pulse increase as the potential becomes more positive. At emission potentials lower than $+1.44 \mathrm{~V}$ the $n$ polarons are in great excess in the polymer and the quenching of the excited stated $S_{1}$ is the dominant process, driving to low intensity of the $\mathrm{AE}$, when a photogenerated singlet exciton $\left(S_{l}\right)$ finds a quencher $(p)$, the exciton-polaron annihilation process indicated in equation 5 takes place:

$$
S_{1}+p \stackrel{k_{q}}{\rightarrow} S_{0}+p+\text { phonons }
$$


Greater AE intensity is obtained from $+1.44 \mathrm{~V}$ since of $p$ polarons that are generated in higher amount. When the anodic potential exceeds $+1.64 \mathrm{~V}$ oxidative degradation processes of the polymer drives to an irreversible loss of the emission.

The intensity of the emission depends not only on the potential of the emission, but also on the potential employed in the cathodic charging. Fig. 4 shows the AE at $1.24 \mathrm{~V}$ after the application of different charging potentials. After a period with no emission (dark period), the ECL emission starts to grow until reaching a maximum intensity and then the emission decays with a characteristic half-life time. The intensity of the emission, the dark period and the half-lives recorded are dependent on the charging potential.
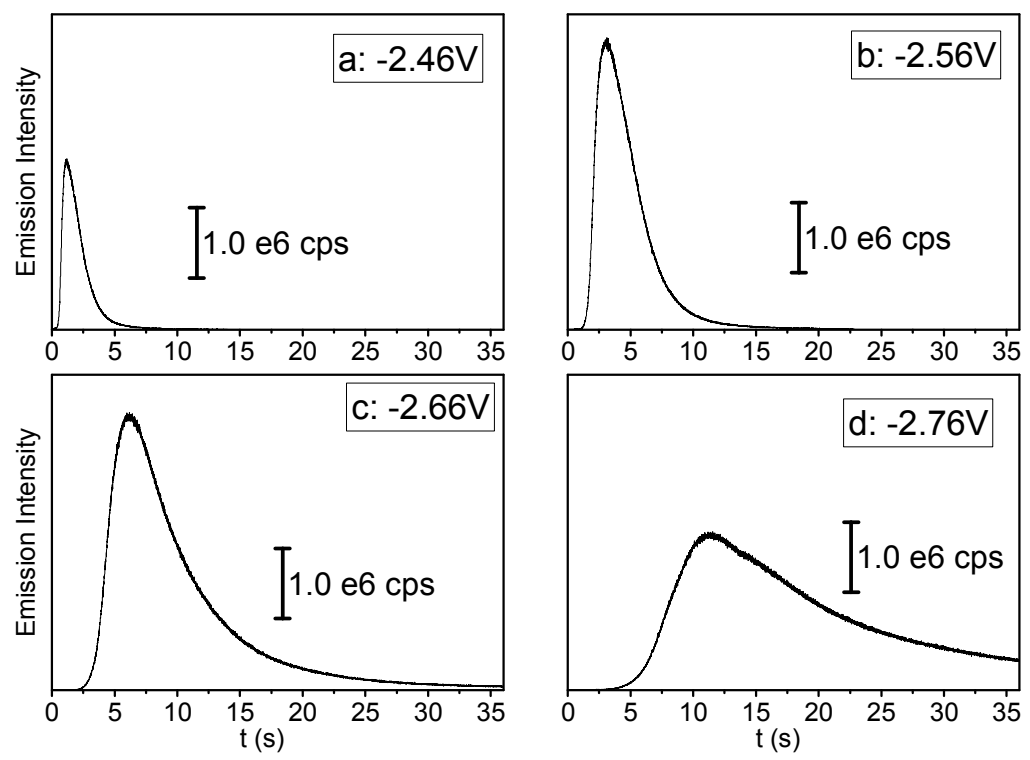

Figure 4.: Anodic emission at $+1.24 \mathrm{~V}$ after charge saturation at different cathodic potentials. The charging potential is indicated in each graph. 
The AE maximum intensity of the emitted light is represented as function of the cathodic charging potential in figure 5 . The maximum intensity depends on the charging potential, it grows from $-2.36 \mathrm{~V}$ (near the reduction onset) until reaching a maximum at $-2.56 \mathrm{~V}$. The imposition of charging potentials more negative than $-2.6 \mathrm{~V}$ produces a lowering in the emission intensity.

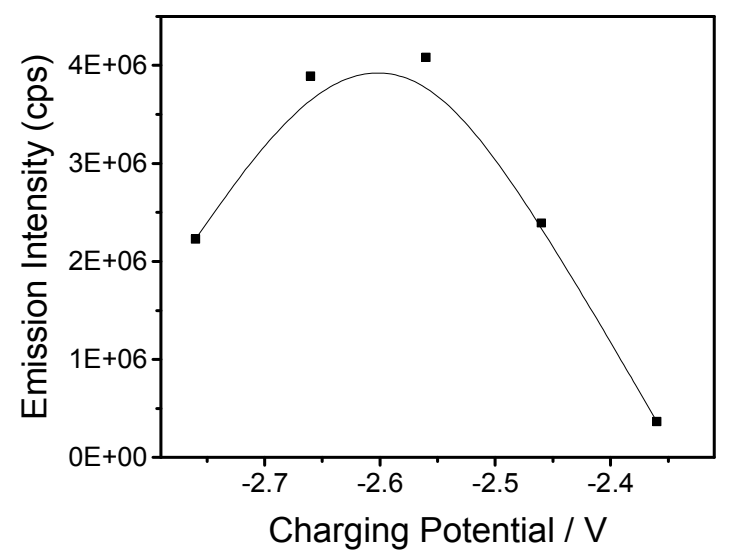

Figure 5: Maximum intensity of the emitted light at $440 \mathrm{~nm}$ in $\mathrm{AE}$ at $+1.24 \mathrm{~V}$ as a function of the cathodic charging potential.

Figure 6 shows the relation between the charging potential and the characteristic times of the emission process: the dark period and the half-life time. In general terms, as the charging potentials becomes more negative we can observe longer dark periods and longer half-lives of emission are observed. The variation of the dark period with the charging potential (fig. 6a) can be explained in terms of quenching of excitons by an excess of $n$ polarons or by the generation of bipolaronic species at highly negative potentials that is detrimental to ECL. 

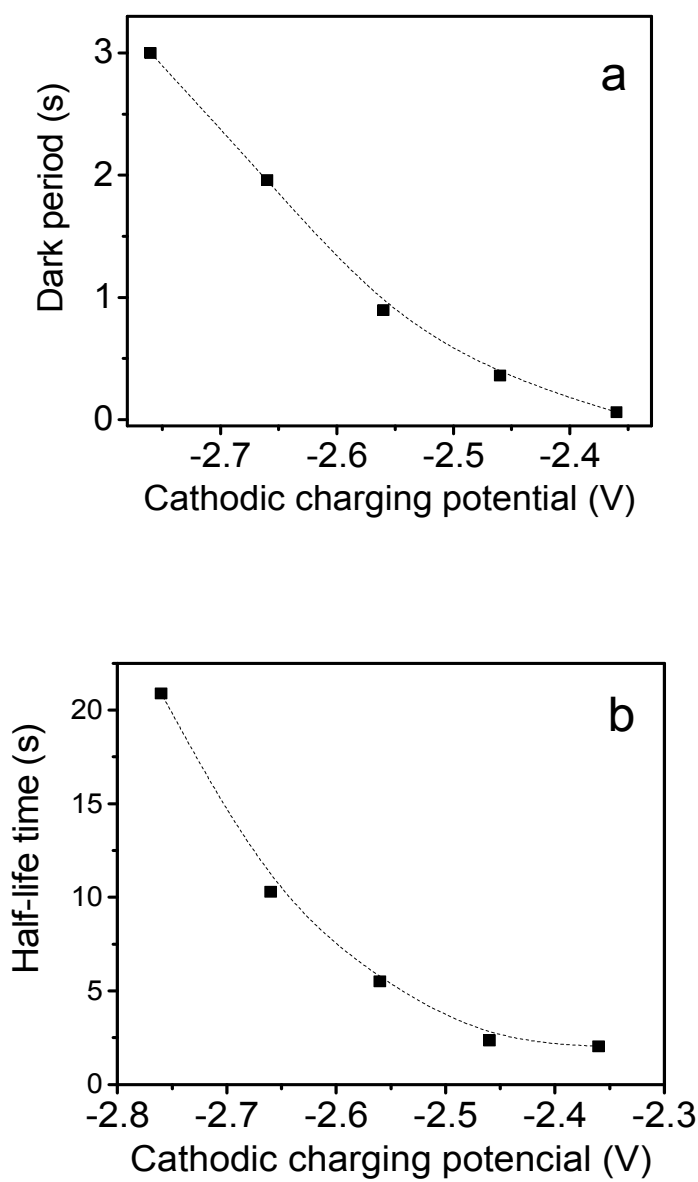

Figure 6: (a) Dark period measured after the anodic pulse at $+1.24 \mathrm{~V}$ as a function of the cathodic charging potential; (b) half-life time of the anodic emission at $+1.24 \mathrm{~V}$ as a function of the cathodic charging potential.

The concentration and ubication of $n$ polaronic (polarons or bipolarons) in the film depends on the cathodic charge potential. The electrochemical behavior of doped conjugated polymers can be rationalized making use of the so-called "oligomer approach" ${ }^{51-53}$. The polymer is composed of different segments with different effective 
conjugated lengths, and generally, the doping states are formed at potentials closer to the doping onset (less negative potentials, in the case of $n$ doping) for the longest conjugated lengths. Therefore, charge injection at deep negative potentials produces the doping of polymer chains with shorter conjugation lengths. ${ }^{54}$ The increase of the dark time at high doping levels during the charging stage is related to larger quenching efficiency of these polarons placed in shorter conjugated segments. In situ electrochemical fluorescence experiments have demonstrated that the introduction of electrochemically injected polarons promotes nonradiative ways for de-excitation of conjugated polymer, as indicated in equation $5^{55,56}$. At low doping levels, the annihilation rate constant, $k_{q}$, depends on the diffusion rate of the species implied in the annihilation process, excitons and quenchers ${ }^{57}$. However at high-doping levels, the exciton-polaron annihilation is dominated by resonant energy transfer via dipole-dipole interaction thus producing a better efficiency of the quenching process. ${ }^{58}$

After the consumption of the excess of $n$ polarons, electrogenerated excitons are formed from the recombination of balanced concentrations of polarons with opposite sign, producing a maximum emission intensity. During the emission, $n$ polarons are completely consumed. In summary, the data of the anodic emission presented in figures 3 and 5 indicate that the optimum potentials for obtaining a maximum anodic emission intensity corresponds to an emission potential of $+1.44 \mathrm{~V}(+0.42 \mathrm{~V}$ above the oxidation onset) and a charging potential of $-2.56 \mathrm{~V}(-0.46 \mathrm{~V}$ below the reduction onset).

\subsection{Cathodic emission}

A similar study to that presented in the previous section has been performed in the emission obtained in cathodic steps (CE). In this case the polymer is initially oxidized 
by applying a potential more positive than the $p$-doping onset $(\mathrm{E}>1.02 \mathrm{~V})$ to proceed with the injection of holes until charge saturation is reached, as indicated in equation 6 :

$$
S_{0}+h^{+} \rightarrow p^{+}(\text {saturation })
$$

Then, the potential was stepped to a cathodic value for the electrochemical injection of electrons and the subsequent emission was collected. Examples of ECL transients are shown in figure S2 and S3 of the supporting information. Similarly, to the case of the AE, a dark period is observed during which no emission is detected. Then, ECL emission starts to grow until reaching a maximum intensity. As in the previous experiments, the shape of the ECL transients is very complex but, in general terms, the radiative decay times are shorter during $\mathrm{CE}$ than the equivalent experiments presented of $\mathrm{AE}$.

We have performed experiments to determine the optimal potentials for a maximum intensity. The emission intensity is presented in figure 7 as a function of the cathodic emission and the anodic charging potentials. Figure 7.a shows the intensity of the maximum emission as function of the cathodic step. As observed, the emission intensity is low at $-2.36 \mathrm{~V}$ (near the reduction onset) but it increases when moving to more negative potentials until reaching a maximum emission at $-2.56 \mathrm{~V}$. The intensity of this emission remains at similar values for more negative potentials (up to $-2.86 \mathrm{~V}$ ). Measurements taken at potentials more negative than $-2.86 \mathrm{~V}$ produce rapid degradation of the polymer.

Figure 7.b shows the effect of the charging potential in the intensity of the cathodic emission. As observed, the intensity increases until reaching a maximum intensity for an anodic charging potential of $+1.44 \mathrm{~V}$. The application of charging potentials more positive than $+1.44 \mathrm{~V}$ produce a decrease of the emission intensity, indicating that 
polaronic species (polarons and bipolarons) are formed in great excess. Charging potentials more positive than $+1.64 \mathrm{~V}$ produce the degradation of the polymer.
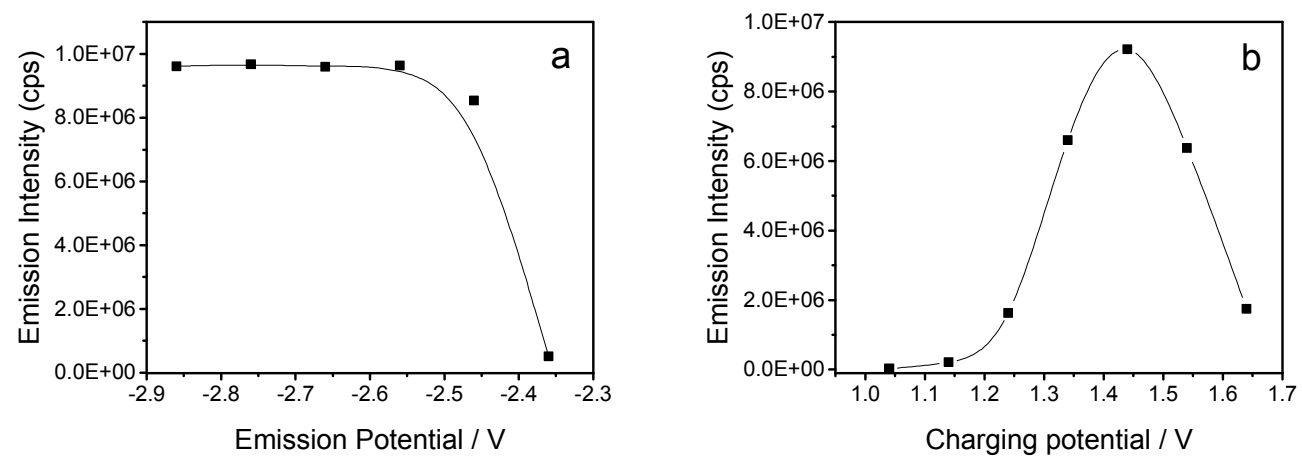

Figure 7: (a) Intensity of the maximum emitted light in cathodic pulses (Cathodic Emission, CE). at different emission potentials. Charging potential +1.24 V. (b) Intensity of the maximum emitted light in $\mathrm{CE}$ at $-2.36 \mathrm{~V}$ as a function of the charging potential.

In summary, the data of the cathodic emission presented in figure 7 , indicate that the optimum potentials for obtaining a maximum emission intensity correspond to an emission potential of $-2.56 \mathrm{~V}$ and a charging potential of +1.44 . These values are symmetrical to those obtained in the anodic emission experiments.

Dark periods are displayed in the figure 8 as a function of the anodic charge potential. As in the previous section dark periods are closely related to the potential reached in the charging stage. 


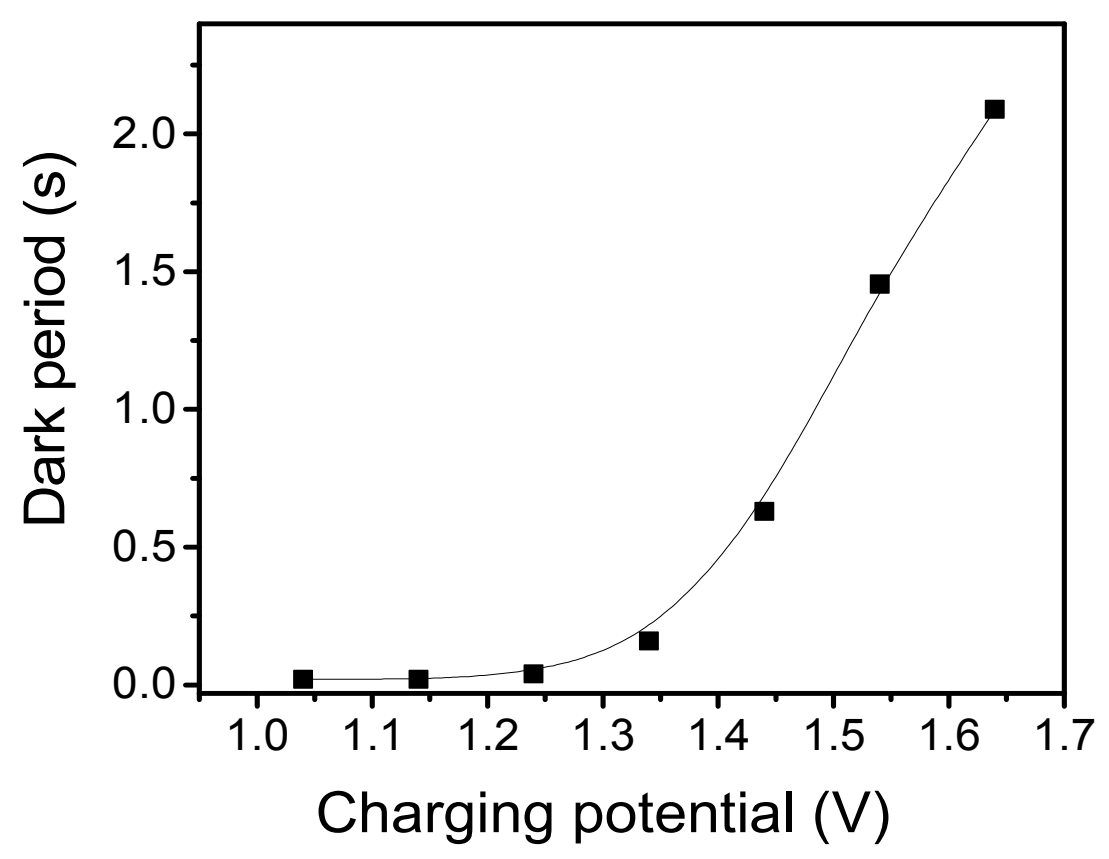

Figure 8. Dark period of cathodic emission at $-2.20 \mathrm{~V}$ as a function of the anodic charging potential.

\begin{abstract}
At moderate charging potential (lesser than $+1.24 \mathrm{~V}$ ) the dark times are close to 0 . Higher potentials drive to longer dark period that increases linearly with the charging potential. This behavior resembles the one presented in the previous section and confirms that the electrochemically generated excitons are initially quenched by the excess of $p$ polarons. After partial depletion of $p$ polarons, the emission coming from the recombination of balanced polarons of opposite sign is observed.

This behavior is like that obtained in the anodic emission, although some subtle differences can be observed, related to different mobilities of holes and electrons within the film. It should be noted that the slope of the curve in fig. 6a measured in the zone of


deep potentials is $10 \mathrm{~s} \mathrm{~V}^{-1}$ while the slope in the present case is $7 \mathrm{~s} \mathrm{~V}^{-1}$ (figure 8). For this two cases quenching is produced by an excess of $n$ and $p$ polarons, respectively. This indicates that $p$ polarons seem to be less efficient quenchers for electrogenerated excitons in these experiments.

Considering the relative mobility of charge carriers in PFO, the hole mobility is in the order of $10^{-2}-10^{-4} \mathrm{~cm}^{2} \mathrm{~V}^{-1} \mathrm{~s}^{-1}$ while the electron mobility is near three orders of magnitude lower, ${ }^{59,60}$ it is reasonable to consider the electrons as fixed charges and the holes as the sole mobile charges in PFO. Hence, the anodic emission would result from recombination of the holes that are injected from the electrode-polymer interface and their movement towards the electrolyte-polymer interface to recombine with the fixed electrons. In that manner, initially the excitons would be mainly generated in the electrode surface and the emission would appear from the electrode surface to moving the electrolyte-polymer interface (see Scheme 1 of the supporting information). This phenomenon is similar to that observed in $\mathrm{p}-\mathrm{n}$ junction of planar light-emitting electrochemical cells. The $p$ doping propagates at a faster speed than $n$ doping. ${ }^{61}$ The propagation of $p$ - and $n$-doping front leads to the formation of the light-emitting $p$ - $n$ junction near the cathode.

On the other hand, the cathodic emission results from the recombination of holes moving from the polymer bulk towards the electrode-polymer interface where fixed electrons are injected. The first quenching process of the generated excitons after transport and polaron recombination should take place in excess of $p$ polarons. After depletion of these polarons, the radiative decay of the excitons can be quenched by excess of $n$ polarons until the saturation by negative charges. The recombination would 
occur near the electrode-polymer interface, so the local concentration of negative polarons nearby electrogenerated excitons would induce a more efficient quenching. Conversely during anodic emission holes are injected from the electrode-polymer interface in a polymer containing electrons. These holes move towards the electrolytepolymer interface finding electrons, that we can considered as fixed charges. In that manner, initially the excitons would be mainly generated near the electrode surface and the emission front will move from the electrode surface to the electrolyte-polymer interface (see Scheme 1 in the supporting information)

\subsection{ECL spectra and stability}

Figure 9 shows the ECL spectra obtained in AE and CE when the optimized potentials were set to $-2.56 \mathrm{~V}$ and $+1.44 \mathrm{~V}$. Both spectra show a vibronic progression and clearly resolved emission with peaks at 441, 469 and 494-496 $\mathrm{nm}$. As observed, the shape of the emission spectrum is similar for both $\mathrm{AE}$ and $\mathrm{CE}$. For comparison purposes, the PL spectrum was included in figure 9. The small widening observed at around $423 \mathrm{~nm}$ is attributed to glassy phase emission. It is known that in PL emission from PFO efficient excitation energy transfer from glassy to the $\beta$-phase domains occur. ${ }^{45,21}$ The close coincidence of the shapes between ECL and PL spectra indicate that ECL originates mainly from the same emissive species, i.e., the $\beta$-phase PFO singlet exciton.

Furthermore, in the ECL spectra, the broad peak at $423 \mathrm{~nm}$ is not observed, indicating a lack of emission from glassy phases. Previous studies performed by spectroelectrochemical methods have shown that polarons are preferentially injected in the $\beta$-phases. ${ }^{62}$ The different relative intensities of the PL and ECL emission peaks might be due to the different behavior of optically and electrically generated emission in 
the PFO films. ${ }^{25}$ The exciton decay in PL can be different to the polaron recombination zones in ECL. In the PL emission, the excitons are optically generated in a wide area of the PFO film, whereas during the ECL the emission depends on the electron and hole relative mobilities, as discussed previously. The latter may explain the different intensity of the peaks centered at 494 and $496 \mathrm{~nm}$ corresponding to $\mathrm{AE}$ and $\mathrm{CE}$, respectively.

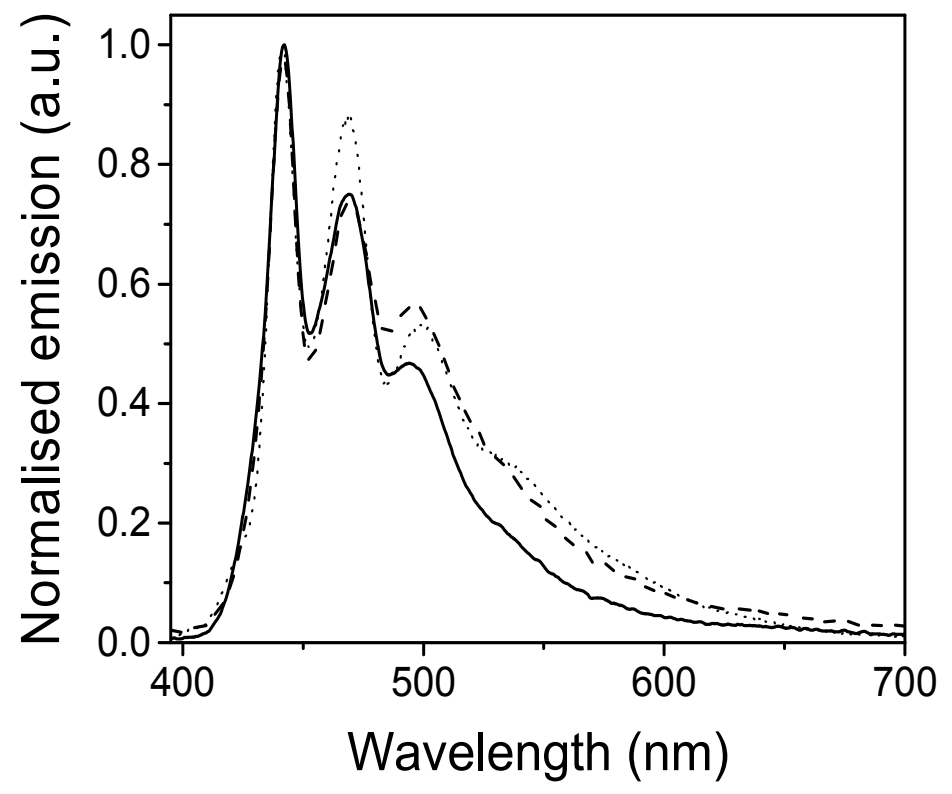

Figure 9. ECL spectra of anodic emission (solid line), cathodic emission (dashed line) and PL spectrum (dotted line) measured for PFO thin films. The ECL spectra were obtained applying potentials of $-2.56 \mathrm{~V}$ and $+1.44 \mathrm{~V}$.

The ECL stability was investigated by recording the temporal evolution of the ECL emission obtained for a PFO film (see figure 10). A potential sequence composed of a 
first step from an initial potential of $0 \mathrm{~V}$ to $+1.44 \mathrm{~V}$ for $1 \mathrm{~s}$ followed by a step to a potential of $-2.56 \mathrm{~V}$ for $1 \mathrm{~s}$.
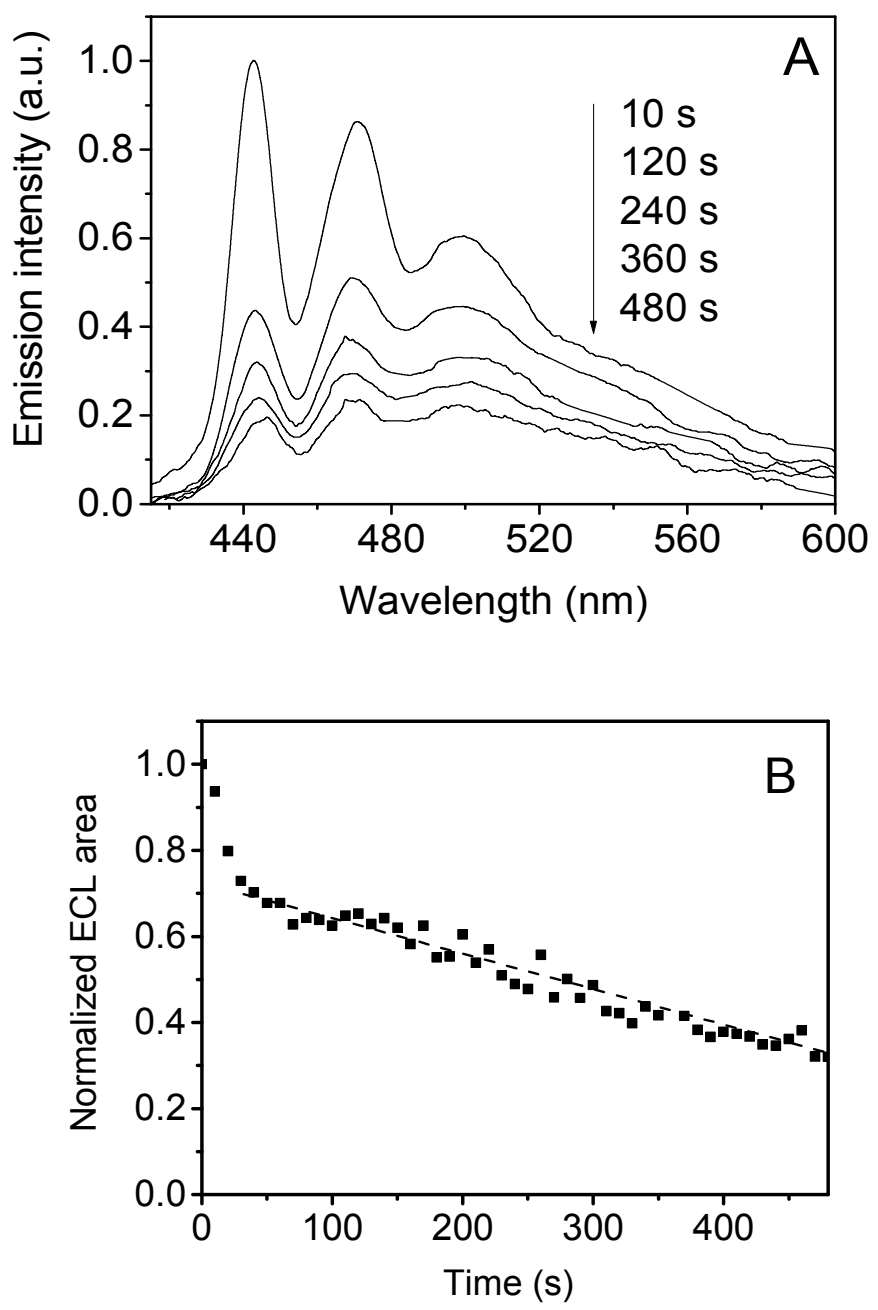

Figure 10. A) ECL spectra measured for a PFO thin film with an integration time of 10 $\mathrm{s}$. The potential is stepped between $+1.44 \mathrm{~V}$ for $1 \mathrm{~s}$ and $-2.56 \mathrm{~V}$ during $1 \mathrm{~s}$. The ECL spectra shown were obtained at different times indicated in the figure. B) Normalized ECL area versus time for all spectra recorded. 
The ECL spectra obtained are averages between $\mathrm{AE}$ and CE, since the integration time used to record the spectra is $10 \mathrm{~s}$. As time grows the ECL emission intensity decrease and the relationship between the intensities of the emission peaks change. This is due to the electrochemical degradation of the polymer chains. Emission peak wavelengths do not substantially change over the time, indicating that the ECL emission originates from the same phases of PFO during the whole period of measurements.

The widening of the spectra observed between 500 and $550 \mathrm{~nm}$ over time can be attributed to the formation of new fluorophores because of the electrochemical degradation.

To quantify the ECL stability, the integrated emission has been plotted as a function of the time in figure 10B. The ECL integrated emission decays rapidly in the first minute to reach an intensity of around $60 \%$ of its initial value. This rapid loss of emission in the initial stages might be explained in terms of dissolution of low molecular weight polymer fragments, that become soluble upon electrochemical doping. The emission spectrum shows a major modification in this period (fig. 10A), the intensity of the main emission peak at $441 \mathrm{~nm}$ decreases with respect to those of the vibronic features. Then, the emission starts to decrease slowly until it vanishes completely after 18 min. During all this period, the spectral shape of the spectra remains stable until the complete vanishing of the emission.

\section{Conclusions}


This study focuses on the optimization of electrochemically-generated chemiluminescence of polyfluorene films obtained by the successive injection of polarons with opposite sign in PFO films. Both, cathodic and anodic ECL emission, were optimized as a function of the emission and charging potentials. The maximum emission intensity was obtained when potentials of $0.4 \mathrm{~V}$ beyond the doping onset of the polymer was applied. Results have shown that while ECL emission is slightly affected by the applied potential, it is strongly affected by the charging potential.

The application of large potentials for charging produce a high concentration of polaronic species (polarons and bipolarons) that are in detrimental of ECL. These species are considered as quenching sites for electrochemically generated excitons. The ECL spectral shape of PFO indicates that the emission occurs mainly from the $\beta$-phase domains in which the electrochemical charge is preferentially injected.

\section{Supporting Information.}

- Cell design for ECL measurement.

- ECL experiments from cathodic emission.

- Scheme of recombination processes during cathodic and anodic emission.

\section{Acknowledgments}

Authors acknowledge financial support from Spanish Ministry of Economy Explora Ciencia Project MAT2013-49534-EXP. 


\section{References}

(1) Yap, B. K.; Xia, R. D.; Campoy-Quiles, M.; Stavrinou, P. N.; Bradley, D. D. C. Simultaneous Optimization of Charge-Carrier Mobility and Optical Gain in Semiconducting Polymer Films. Nat. Mater. 2008, 7, 376-380.

(2) Tsiminis, G.; Ruseckas, A.; Samuel, I. D. W.; Turnbull, G. A. A Two-Photon Pumped Polyfluorene Laser. Appl. Phys. Lett. 2009, 94.

(3) Ryu, G.; Stavrinou, P. N.; Bradley, D. D. C. Spatial Patterning of the Beta-Phase in Poly (9,9-Dioctylfluorene): A Metamaterials-Inspired Molecular Conformation Approach to the Fabrication of Polymer Semiconductor Optical Structures. Adv. Funct. Mater. 2009, 19, 3237-3242.

(4) Yang, Y.; Turnbull, G. A.; Samuel, I. D. W. Hybrid Optoelectronics: A Polymer Laser Pumped by a Nitride Light-Emitting Diode. Appl. Phys. Lett. 2008, 92.

(5) Yang, Y.; Turnbull, G. A.; Samuel, I. D. W.; Samuel, D. W. Sensitive Explosive Vapor Detection with Polyfluorene Lasers. Adv. Funct. Mater. 2010, 20, $2093-$ 2097.

(6) Anni, M.; Rella, R. Oxygen Optical Gas Sensing by Reversible Fluorescence Quenching in Photo-Oxidized poly(9,9-Dioctylfluorene) Thin Films. J. Phys. Chem. B 2010, 114, 1559-1561.

(7) Chou, C.-H.; Yang, C.-H.; Hsu, C.-S.; Chen, T.-M. Hybrid White-Light Emitting-LED Based on Luminescent Polyfluorene Polymer and Quantum Dots. J. Nanosci. Nanotechnol. 2007, 7, 2785-2789.

(8) Zhao, Z.; Li, J. H.; Lu, P.; Yang, Y. Fluorescent, Carrier-Trapping Dopants for 
Highly Efficient Single-Layer Polyfluorene LEDs. Adv. Funct. Mater. 2007, 17, $2203-2210$.

(9) Vasilopoulou, M.; Palilis, L. C.; Botsialas, A.; Georgiadou, D. G.; Bayiati, P.; Vourdas, N.; Petrou, P. S.; Pistolis, G.; Stathopoulos, N. A.; Argitis, P. Flexible Organic Light Emitting Diodes (OLEDs) Based on a Blue Emitting Polyfluorene. In Physica Status Solidi (C) Current Topics in Solid State Physics; 2008; Vol. 5, pp 3658-3662.

(10) Na, S. I.; Oh, S. H.; Kim, S. S.; Kim, D. Y. Efficient Organic Solar Cells with Polyfluorene Derivatives as a Cathode Interfacial Layer. Org. Electron. physics, Mater. Appl. 2009, 10, 496-500.

(11) Chen, M. H.; Hou, J.; Hong, Z.; Yang, G.; Sista, S.; Chen, L. M.; Yang, Y. Efficient Polymer Solar Cells with Thin Active Layers Based on Alternating Polyfluorene Copolymer/fullerene Bulk Heterojunctions. Adv. Mater. 2009, 21, $4238-4242$.

(12) Zhu, Z.; Bai, Y.; Lee, H. K. H.; Mu, C.; Zhang, T.; Zhang, L.; Wang, J.; Yan, H.; So, S. K.; Yang, S. Polyfluorene Derivatives Are High-Performance Organic Hole-Transporting Materials for Inorganic-Organic Hybrid Perovskite Solar Cells. Adv. Funct. Mater. 2014, 24, 7357-7365.

(13) Bradley, D. D. C.; Grell, M.; Long, X.; Mellor, H.; Grice, A.; Road, H.; Inbasekaran, M.; Woo, E. P. Influence of Aggregation on the Optical Properties of a Polyfluorene. Proc. SPIE 1997, 3145, 254-259.

(14) Chunwaschirasiri, W.; Tanto, B.; Huber, D. L.; Winokur, M. J. Chain Conformations and Photoluminescence of Poly(di-N-Octylfluorene). Phys. Rev. 


\section{Lett. 2005, 94.}

(15) Chen, S. H.; Su, A. G.; Su, C. H.; Chen, S. A. Crystalline Forms and Emission Behavior of poly(9,9-Di-N-Octyl-2,7-Fluorene). Macromolecules 2005, 38, 379385.

(16) Chen, S. H.; Su, A. C.; Chen, S. A. Noncrystalline Phases in poly(9,9-Di-NOctyl-2,7-Fluorene). J. Phys. Chem. B 2005, 109, 10067-10072.

(17) Grell, M.; Bradley, D. D. C.; Inbasekaran, M.; Woo, E. P. A Glass-Forming Conjugated Main-Chain Liquid Crystal Polymer for Polarized Electroluminescence Applications. Adv. Mater. 1997, 9, 798-802.

(18) Grell, M.; Bradley, D. D. C.; Ungar, G.; Hill, J.; Whitehead, K. S. Interplay of Physical Structure and Photophysics for a Liquid Crystalline Polyfluorene. Macromolecules 1999, 32, 5810-5817.

(19) Grell, M.; Bradley, D. D. C.; Long, X.; Chamberlain, T.; Inbasekaran, M.; Woo, E. P.; Soliman, M. Chain Geometry, Solution Aggregation and Enhanced Dichroism in the Liquid-Crystalline Conjugated Polymer poly(9,9Dioctylfluorene). Acta Polym. 1998, 49, 439-444.

(20) Liu, C.; Wang, Q.; Tian, H.; Liu, J.; Geng, Y.; Yan, D. Morphology and Structure of the $\beta$ Phase Crystals of Monodisperse Polyfluorenes. Macromolecules 2013, 46, 3025-3030.

(21) Khan, A. L. T.; Sreearunothai, P.; Herz, L. M.; Banach, M. J.; Köhler, A. Morphology-Dependent Energy Transfer within Polyfluorene Thin Films. Phys. Rev. B 2004, 69, 85201. 
(22) Cadby, A. J.; Lane, P. A.; Mellor, H.; Martin, S. J.; Grell, M.; Giebeler, C.;

Bradley, D. D. C.; Wohlgenannt, M.; An, C.; Vardeny, Z. V. Film Morphology and Photophysics of Polyfluorene. Phys. Rev. B 2000, 62, 15604-15609.

(23) Ariu, M.; Lidzey, D. G.; Sims, M.; Cadby, A. J.; Lane, P. A.; Bradley, D. D. C. The Effect of Morphology on the Temperature-Dependent Photoluminescence Quantum Efficiency of the Conjugated Polymer poly(9, 9-Dioctylfluorene). J. Physics-Condensed Matter 2002, 14, 9975-9986.

(24) Prins, P.; Grozema, F. C.; Nehls, B. S.; Farrell, T.; Scherf, U.; Siebbeles, L. D. A. Enhanced Charge-Carrier Mobility in $\beta$-Phase Polyfluorene. Phys. Rev. B Condens. Matter Mater. Phys. 2006, 74.

(25) Cheun, H.; Rugheimer, P. P.; Larson, B. J.; Gopalan, P.; Lagally, M. G.; Winokur, M. J. Polymer Light Emitting Diodes and Poly(di-N-Octylfluorene) Thin Films as Fabricated with a Microfluidics Applicator. J. Appl. Phys. 2006, 100,73510 .

(26) Rothe, C.; Galbrecht, F.; Scherf, U.; Monkman, A. The Beta-Phase of poly(9,9Dioctylfluorene) as a Potential System for Electrically Pumped Organic Lasing. Adv. Mater. 2006, 18, 2137.

(27) Richter, M. M.; Fan, F. R. F.; Klavetter, F.; Heeger, A. J.; Bard, A. J. Electrochemistry and Electrogenerated Chemiluminescence of Films of the Conjugated Polymer 4-Methoxy-(2-Ethylhexoxyl)-2,5- Polyphenylenevinylene. Chem. Phys. Lett. 1994, 226, 115-120.

(28) Forster, R. J.; Bertoncello, P.; Keyes, T. E. Electrogenerated Chemiluminescence. Annu. Rev. Anal. Chem. 2009, 2, 359-385. 
(29) Puodziukynaite, E.; Oberst, J. L.; Dyer, A. L.; Reynolds, J. R. Establishing Dual Electrogenerated Chemiluminescence and Multicolor Electrochromism in Functional Ionic Transition-Metal Complexes. J. Am. Chem. Soc. 2012, 134, 968-978.

Omer, K. M.; Ku, S.-Y.; Chen, Y.-C.; Wong, K.-T.; Bard, A. J. Electrochemical Behavior and Electrogenerated Chemiluminescence of Star-Shaped D-A Compounds with a 1,3,5-Triazine Core and Substituted Fluorene Arms. J. Am. Chem. Soc. 2010, 132, 10944-10952.

(31) Doeven, E. H.; Barbante, G. J.; Kerr, E.; Hogan, C. F.; Endler, J. A.; Francis, P. S. Red-Green-Blue Electrogenerated Chemiluminescence Utilizing a Digital Camera as Detector. Anal. Chem. 2014, 86, 2727-2732.

(32) Valenti, G.; Fiorani, A.; Li, H.; Sojic, N.; Paolucci, F. Essential Role of Electrode Materials in Electrochemiluminescence Applications. ChemElectroChem 2016, 3, 1990-1997.

(33) Valenti, G.; Rampazzo, E.; Bonacchi, S.; Petrizza, L.; Marcaccio, M.; Montalti, M.; Prodi, L.; Paolucci, F. Variable Doping Induces Mechanism Swapping in Electrogenerated Chemiluminescence of $\mathrm{Ru}(\mathrm{bpy})_{3}{ }^{2+}$ Core-Shell Silica Nanoparticles. J. Am. Chem. Soc. 2016, 138, 15935-15942.

(34) Chen, J. T.; Chang, Y. L.; Guo, S.; Fabian, O.; Lackowski, W. M.; Barbara, P. F. Electrogenerated Chemiluminescence of Pure Polymer Films and Polymer Blends. Macromol. Rapid Commun. 2011, 32, 598-603.

Chen, J.-T.; Chang, Y.-L.; Fabian, O.; Guo, S.; Lackowski, W. M.; Barbara, P. F. Effects on Oxidation Waves of Conjugated Polymers by Studying 
Photoluminescence Quenching and Electrogenerated Chemiluminescence. $J$. Phys. Chem. C 2011, 115, 10256-10263.

(36) Chang, Y. L.; Palacios, R. E.; Chen, J. T.; Stevenson, K. J.; Guo, S.; Lackowski, W. M.; Barbara, P. F. Electrogenerated Chemiluminescence of Soliton Waves in Conjugated Polymers. J. Am. Chem. Soc. 2009, 131, 14166.

(37) Dini, D.; Martin, R. E. E.; Holmes, A. B. B. Anodic and Cathodic Electrochemically Generated Chemiluminescence in Conjugated Polymers. Adv. Funct. Mater. 2002, 12, 299-306.

(38) Montilla, F.; Mallavia, R. On the Origin of Green Emission Bands in FluoreneBased Conjugated Polymers. Adv. Funct. Mater. 2007, 17, 71-78.

(39) Measures, R. M. Prospects for Developing a Laser Based on Electrochemiluminescence. Appl. Opt. 1974, 13, 1121.

(40) Keszthelyi, C. P. Laser Based on Electrochemiluminescence. Appl. Opt. 1975, 14,1710 .

(41) Horiuchi, T.; Niwa, O.; Hatakenaka, N. Evidence for Laser Action Driven by Electrochemiluminescence. 1998, 394, 659-661.

(42) Connelly, N. G.; Geiger, W. E. Chemical Redox Agents for Organometallic Chemistry. Chem. Rev. 1996, 96, 877-910.

(43) Montilla, F.; Ruseckas, A.; Samuel, I. D. W. Absorption Cross-Sections of Hole Polarons in Glassy and $\beta$-Phase Polyfluorene. Chem. Phys. Lett. 2013, 585, 133137.

(44) Bansal, A. K.; Ruseckas, A.; Shaw, P. E.; Samuel, I. D. W. Fluorescence 
Quenchers in Mixed Phase Polyfluorene Films. J. Phys. Chem. C 2010, 114, $17864-17867$.

Ariu, M.; Sims, M.; Rahn, M. D.; Hill, J.; Fox, A. M.; Lidzey, D. G.; Oda, M.;

Cabanillas-Gonzalez, J.; Bradley, D. D. C. Exciton Migration in $\beta$-Phase poly(9,9-Dioctylfluorene). Phys. Rev. B 2003, 67, 195333.

(46) Gamerith, S.; Gaal, M.; Romaner, L.; Nothofer, H.-G. G.; Güntner, R.;

Scandiucci de Freitas, P.; Scherf, U.; List, E. J. W. J. W.; Guntner, R.; de Freitas, P. S.; et al. Comparison of Thermal and Electrical Degradation Effects in Polyfluorenes. Synth. Met. 2003, 139, 855-858.

(47) Becker, K.; Lupton, J. M. M.; Feldmann, J.; Nehls, B. S. S.; Galbrecht, F.; Gao, D. Q.; Scherf, U. On-Chain Fluorenone Defect Emission from Single Polyfluorene Molecules in the Absence of Intermolecular Interactions. $A d v$.

Funct. Mater. 2006, 16, 364-370.

(48) Chan, K. L.; Sims, M.; Pascu, S. I.; Ariu, M.; Holmes, A. B.; Bradley, D. D. C. Understanding the Nature of the States Responsible for the Green Emission in Oxidized Poly(9,9-Dialkylfluorene)s: Photophysics and Structural Studies of Linear Dialkylfluorene/Fluorenone Model Compounds. Adv. Funct. Mater. 2009, $19,2147-2154$.

(49) Nikitenko, V. R.; Kadashchuk, A.; Schmechel, R.; von Seggern, H.; Korosko, Y. Effect of Dispersive Transport and Partial Trap Filling on Thermally Stimulated Current in Conjugated Polymers. J. Appl. Phys. 2005, 98, 103702.

(50) Yang, C.; He, G.; Wang, R.; Li, Y. Solid-State Electrochemical Investigation of poly[2-Methoxy, 5-(2'-Ethyl-Hexyloxy)-1,4-Phenylene Vinylene]. $J$. 
Electroanal. Chem. 1999, 471, 32-36.

(51) Elandaloussi, E. H.; Frère, P.; Richomme, P.; Orduna, J.; Garin, J.; Roncali, J. Effect of Chain Extension on the Electrochemical and Electronic Properties of $\pi$ Conjugated Soluble Thienylenevinylene Oligomers. J. Am. Chem. Soc. 1997, 119, 10774-10784.

(52) Heinze, J.; Tschuncky, P.; Smie, A. The Oligomeric Approach - the Electrochemistry of Conducting Polymers in the Light of Recent Research. $J$. Solid State Electrochem. 1998, 2, 102-109.

(53) Heinze, J.; Frontana-Uribe, B. a.; Ludwigs, S. Electrochemistry of Conducting Polymers-Persistent Models and New Concepts. Chem. Rev. 2010, 110, 47244771 .

(54) Chi, C. Y.; Wegner, G. Chain-Length Dependence of the Electrochemical Properties of Conjugated Oligofluorenes. Macromol. Rapid Commun. 2005, 26, $1532-1537$.

(55) Montilla, F.; Mallavia, R. In Situ Electrochemical Fluorescence Studies of PPV. J. Phys. Chem. B 2006, 110, 25791-25796.

(56) Montilla, F.; Frutos, L. M.; Mateo, C. R.; Mallavia, R. Fluorescence Emission Anisotropy Coupled to an Electrochemical System: Study of Exciton Dynamics in Conjugated Polymers. J. Phys. Chem. C 2007, 111, 18405-18410.

(57) Lakowicz, J. R. Principles of Fluorescence Spectroscopy; Plenum Press: New York, 2013.

(58) Montilla, F.; Huerta, F. Electrochemically Monitored Photoluminescence of 
Conjugated Polymers. In Luminescence in Electrochemistry; Springer

International Publishing: Cham, 2017; pp 105-137.

(59) Yang, C. M.; Liao, H. H.; Horng, S. F.; Meng, H. F.; Tseng, S. R.; Hsu, C. S.

Electron Mobility and Electroluminescence Efficiency of Blue Conjugated

Polymers. Synth. Met. 2008, 158, 25-28.

(60) Pinner, D. J.; Friend, R. H.; Tessler, N. Transient Electroluminescence of

Polymer Light Emitting Diodes Using Electrical Pulses. J. Appl. Phys. 1999, 86, $5116-5130$.

(61) Hu, Y.; Tracy, C.; Gao, J. High-Resolution Imaging of Electrochemical Doping and Dedoping Processes in Luminescent Conjugated Polymers. Appl. Phys. Lett. 2006, $88,1-4$.

(62) Montilla, F.; Ruseckas, A.; Samuel, I. D. W. Absorption Cross-Sections of Hole Polarons in Glassy and Beta-Phase Polyfluorene. Chem. Phys. Lett. 2013, 585, $133-137$. 
TOC Graphic

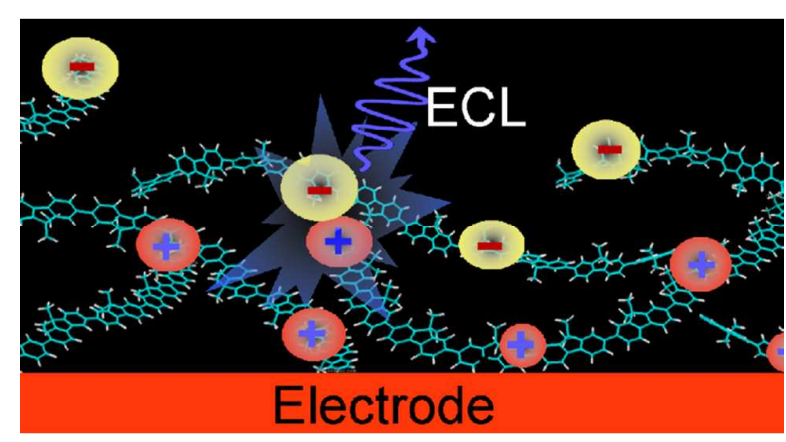

